\title{
PREVALENCE OF FUNGAL KERATITIS FROM TERTIARY CARE HOSPITAL FROM WESTERN PART OF INDIA
}

\author{
JADHAV S.V. ${ }^{*}$, GANDHAM N.R.*, MISRA R.N., UJAGARE M.T., SHARMA M. AND SARDAR M. \\ Pad. Dr. D.Y. Patil Medical College and Research Centre, Pimpri, Pune-18, MS, India. \\ *Corresponding Author: Email- patilsv78@gmail.com
}

Received: April 20, 2012; Accepted: May 03, 2012

\begin{abstract}
-
Introduction: Corneal diseases are a major cause of vision loss and blindness, and caused by bacteria, fungi and protozoa. However, within the topics, as many as two third of ulcer may be due to filamentous fungi and distribution may vary considerably between continents and countries and also within countries. It is essential to determine the local etiology within a given region when planning a corneal ulcer management strategy.

Aim: To identify local etiology for corneal ulceration at a tertiary care hospital in western India during a period of Jan 2007 to Dec 2010. Methods: Patients clinically suspected of microbial keratitis were recruited in the study. Corneal ulceration was defined as a loss corneal epithelium with clinical evidence of infection with or without hypopyon. Microscopy and culture were performed by standard conventional method.

Result: Of 271 patients investigated fungal etiology was established in $68(25.9 \%)$ of which $48(70.58 \%)$ were males. Among the fungal isolates, filamentous fungi, ie 53(77.94\%) were predominant. Among these 24(45.28 \%) were Aspergillus flavus followed by A. fumigates 9 $(16.98 \%)$ and Fusarium spp. 8(15.9\%). Of the 15 isolates of yeasts, Non Candida albicans were identified in 9(60\%) cases.

Conclusion: Infection by filamentous fungi are frequent cause of corneal damage in tropical developing countries and are difficult to treat. Microscopy and culture is an essential tool in the diagnosis of such infections. Knowledge of the "local" etiology within a region is of value in the management of suppurative keratitis.
\end{abstract}

Key words- Fungal Keratitis, Corneal Scraping, Filamentous Fungi, Fungal Culture.

Citation: Jadhav S.V., et. al. (2012) Prevalence of Fungal Keratitis from Tertiary Care Hospital from Western Part of India. International Journal of Microbiology Research, ISSN: 0975-5276 \& E-ISSN:0975-9174, Volume 4, Issue 4, pp.-211-214.

Copyright: Copyright(02012 Jadhav S.V., et. al. This is an open-access article distributed under the terms of the Creative Commons Attribution License, which permits unrestricted use, distribution, and reproduction in any medium, provided the original author and source are credited.

\section{Introduction}

Keratitis is an inflammation of the cornea produced by infectious organisms or non-infectious agents or stimuli. Infectious keratitis is a significant public health problem and caused by bacteria, viruses, fungi, and parasites. Keratitis often causes corneal scarring and opacification. Corneal diseases are major causes of vision loss and blindness [1]. It is estimated that ocular trauma and corneal ulceration result in 1.5 to 2 million new cases of corneal blindness annually and common in outdoor workers and densely populated continent of Asia and Africa. In India, fungal keratitis is more common in warmer climates and rural areas. It often follows trauma to the cornea, especially by organic vegetative matter or object contaminated by soil. Topical application of corticosteroids may predispose to fungal keratitis [2]. Patient's wearing any type of contact lens can develop fungal keratitis. Other disorders, including corneal surface disorders, dry eye, bullous keratopathy, and exposure keratitis are associated with the development of ulcerative keratitis [3]. Generally prompt diagnosis and expedient treatment is helpful in preventing blindness or even enucleation. There are few clinical signs and diversity of clinical presentation observed in each case that can distinguish infectious keratitis from corneal inflammation associated with trauma, hypersensitivity or immune mediated conditions [3, 4]. Diagnosis is associated by the patient's history and ocular examination and confirmed by 
either culture or corneal biopsy. Microbiological tests are needed to establish the etiologic agent and their antimicrobial and/or antifungal susceptibility without which it is difficult to initiate topical antifungal, antibacterial or antiviral treatment. Diagnosis of fungal keratitis is typically occurs late because of misdiagnosis as a bacterial keratitis, clinicians often consider fungal keratitis only after a presumed bacterial keratitis worsens during antibiotic therapy. However, therapy begun before these results is final. The most commonly encountered organisms involved in fungal keratitis show tremendous geographical variation. Prevalence of fungi as a causative agent of fungal keratitis is not just affected geographically but also changes with time, type of weather and individual patient factors which tend to favor specific organisms. Fungal keratitis are frequently caused by filamentous fungi i.e. Fusarium species (37.62\%) and Aspergillus species (24 -30\%). Dematiaceous fungi are the cause of $8-16 \%$ of fungal keratitis $[5,6]$. Yeast can also cause keratitis usually caused by patients own flora. Once there is a clinical suspicion of a fungal infection, every effort should be made to know the fungal etiology to initiate appropriate antifungal therapy. A delay or incorrect treatment could lead to be serious loss of vision or even loss of eye ball. Isolation of fungi from corneal scrapings is essential to confirm diagnosis of fungal keratitis, but requires 48-72 hrs. However, a tentative diagnosis of fungal keratitis can be done by microscopic findings. Low cost tool like direct microscopy by Potassium Hydroxide (KOH 10\%) mount and Gram's staining or Giemsa Staining play a major role in presumptive diagnosis. Lactophenol cotton blue (LPCB) is both a mounting fluid and a stain. This is commonly used in microbiological laboratories for microscopy of fungal culture. It preserves the characteristic morphology and gives colour to the structure to enable clear visualization at the same time kills the agent $[6,7,8]$. As occurrence and spectrum of fungal keratitis varies significantly from country to country and also from region to region, it is obligatory to develop inclusive strategy for diagnosis and treatment of fungal keratitis, so with the aim of that the etiological factors must be determined at various national levels. The rationale of this study was to evaluate prevalence and varying spectrum of fungal keratitis and treatment outcomes amongst patients attending in Pad. Dr. D. Y. Patil Medical College \& Hospital, Pimpri, Pune.

\section{Materials and Methods}

Setting: Pad. Dr. D. Y. Patil Medical College \& Hospital and Research Centre is a 1370 bedded tertiary care hospital in Western part of Maharashtra, India. The time period for this retrospective study was from Jan. 2007 To June 2010.

Study materials: Over a period of four years of the study, we processed corneal scrapings of suspected infectious keratitis patients. By protocol, duplicate isolates from same patients (the same genus and species from the same patients) were excluded. The requisition form was a standard proforma filled up by the ophthalmologist, documenting socio-demographic information, as well as clinical information including duration of symptoms, previous treatment, predisposing ocular conditions and related risk factors. Every patient was examined by an ophthalmologist and corneal scraping was obtained under aseptic conditions by an ophthalmologist. Material was sent to Microbiology laboratory for presumptive identification \& confirmation of causative agent. Material obtained from scraping was inoculated directly on Sabouraud dextrose agar (SDA) with and without antibiotics, i.e, Chloramphenicol $(50 \mathrm{mg} /$ $\mathrm{ml})$ \& Gentamicin $(20 \mathrm{mg} / \mathrm{ml})$ and incubated at $25^{\circ} \mathrm{C} \& 37^{\circ} \mathrm{C}$ separately over a period of four weeks $[8,9,10]$. Sample was also processed by standard conventional methods for the microbial etiology [10]. Materials from corneal scraping was also smeared on two clean grease free glass slides for Gram's staining and 10 $\% \mathrm{KOH}$ wet mount. All material and culture media were obtained from Hi-media Laboratories, Mumbai, India.

\section{Identification of fungal growth}

Fungus identification was done based on the growth rate, colony morphology, reverse and obverse surface colour of SDA slant \& microscopic aspects such as mycelium \& conidia types. Dematiaceous moulds were considered when colonies that can develop dark gray to black mycelium, particularly prominent when a black reverse of the colony was observed.

Identification of hyaline filamentous fungi was done by rapid, flat growth. It was at first white and slightly fuzzy but as the conidia developed it became dark bluish-green and powdery. Old cultures have a grayish "smoky" cast that is quite characteristic of genus Aspergillus [10,11].

Species identification was done by LPCB of culture positive fungi. LPCB revealed microscopic findings as follows ie, inverted flask like shape of vesicle with rounded bottom and long drawn out neck, a single row of sterigmata is borne on the upper half of the vesicle in a more or less crowded parallel formation, conidia are globose, green \& rough - surfaced. Fusarium spp. Are believed to account for at least one third of all reported cases of mycotic keratitis in tropical and subtropical zone. Fusarium solani is the species most frequently isolated from ocular infections. In culture, Fusarium reveal distinctive, septate, large, banana shaped macrocoidia, with apical and basal cells. Genus Colletotrichum have been previously implicated as etiological agent with fungal keratitis. Collectotrichum dematium can be clearly distinguished from other species of Collectotrichum by the occurrence of falcate macroconidia. Yeast identification was done by standard conventional methods like Germ tube test, crease production, chlamydospores production on Corn-meal Agar (CMA) \& species differentiation was supported by phenotypic identification and differentiation on CHROM agar[13,14,15,16].

\section{Results and Observations}

Of 436 cases of culture positive microbial keratitis investigated, a fungal keratitis diagnosis was established in 117 (26.83\%) cases by standard criteria (Figure-1).

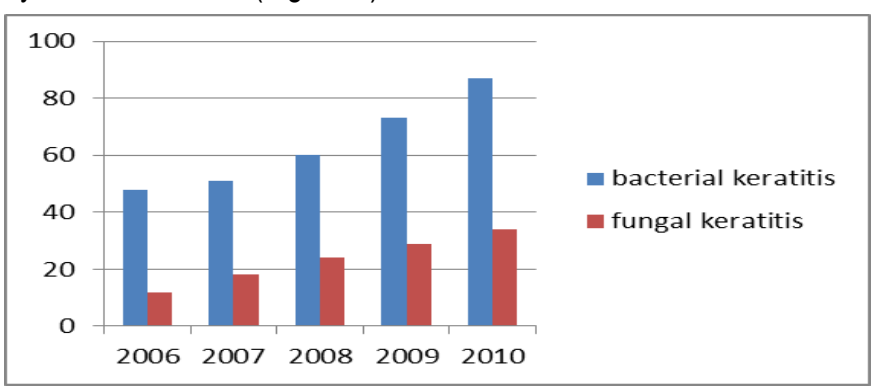

Fig. 1- The annual frequency of positive fungal keratitis 
Of these $88(75.21 \%)$ were male and $29(24.78 \%)$ were female. The most common affected age group was between 31 to $40 \mathrm{yrs}$ in both sexes. Incidence of microbial keratitis is rising every year (Table No.1, Fig.1).Frequency of bacterial keratitis is more as compared to fungal keratitis (Fig.2).

Table 1-The annual frequency of positive fungal keratitis

\begin{tabular}{|lll|}
\hline Year & Bacterial Keratitis & Fungal Keratitis \\
\hline 2006 & 48 & 12 \\
2007 & 51 & 18 \\
2008 & 60 & 24 \\
2009 & 73 & 29 \\
2010 & 87 & 34 \\
TOTAL & 319 & 117 \\
\hline
\end{tabular}

Direct microscopic examination of the $\mathrm{KOH}$ mount of corneal scrapings revealed the presence of fungal hyphae in $88 \%$ of culture positive cases. No fungal element was seen in $95 \%$ of culture negative cases. Likewise direct microscopic examination of corneal scrapings by Gram's stained smear examination showed fungal hyphae and/or yeast like cells in $81 \%$ of culture positive cases. The principal fungus isolated by culture was Aspergillus flavus (24 cases ), followed by Aspergillus fumigates (15cases) \& Fusarium spp. (10 cases ). Dematiaceous fungi were isolated in (8) patients i.e, Alternaria spp. (2 cases ), Curvularia spp. (2 cases ), Cladosporium spp. (3 cases ) and Fonsecaeae solani. (3 cases ), Colletotrichum dematium (2 cases). A total of 30 (25.64\%) cases had yeast infections i.e, Candida infections. Of these 14 cases were revealed Candida non-albicans, i.e, C. parapsilosis (4 cases), C. glabrata (4 cases), C. tropicalis (6 cases) and Candida albicans (16 cases ) [Table 2].

Yeast infection was most commonly seen in cases with history of prior ocular surface disease like dry eyes (4 cases ), allergic eye diseases (2 cases ), prior microbial infections (8 cases), bullous keratopathy (4cases), and recurrent erosion syndrome (1cases). None of the patients were contact lens users among the yeast infection of fungal keratitis. Microbial co-infection was found in 14 cases which were with Pseudomonas aeruginosa (5) Staphylococcus aureus (4), Klebsiella pneumoniae (2), Citrobacter (2) and E. coli (1) infection.

Analysis of the time of occurrence of fungal keratitis revealed higher trends during months of September to November (winter) each year i.e, total 42 cases in four years. 10 cases occurred during month of March to May. 18 cases showed fungal keratitis in the span of four years during rainy season i.e, from June to September. Of the 68 fungal culture positive keratitis 27 cases were agriculturists/farmers, 13 patients gave a history of ocular trauma. 6 patients used topical steroids prior to attend ophthalmic OPD. 17 cases of filamentous hyaline fungal infection developed in contact lens users. 2 patients had diabetes mellitus and Fusarium spp. was isolated from both the cases. Fusarium spp. was isolated from two cases that were on renal dialysis and HIV reactive. The presence of hypopyon was more common in filamentous fungal infections. In three patients, no fungal growth was documented but gram stained smear of corneal scrapings showed fungal elements \& out of these two patients improved clinically on antifungal treatment.

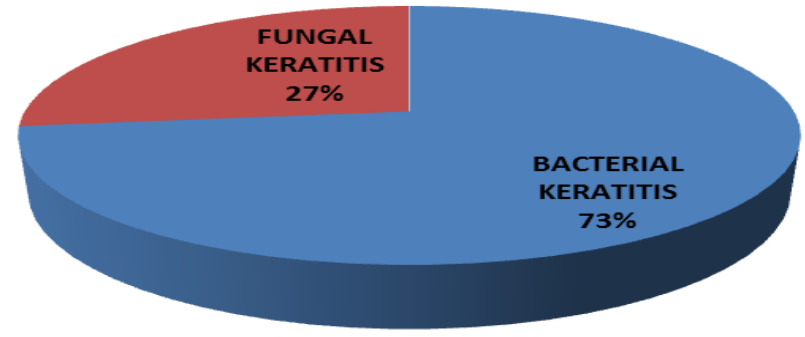

Fig. 2- Distribution of microbial keratitis $n=436$

Table 2- Distriibution of hyaline dematiaceous fungi and yeast in 117 fungal etiology

\begin{tabular}{|c|c|c|c|}
\hline & Fungal etiology & & Total (\%) \\
\hline \multirow[t]{5}{*}{ A. } & \multicolumn{3}{|l|}{ Filamentous Fungi } \\
\hline & \multirow{10}{*}{ Hyaline } & Aspergillus flavus & 24 \\
\hline & & Aspergillus fumigatus & 15 \\
\hline & & Aspergillus terrus & 10 \\
\hline & & Aspergillus nidulans & 8 \\
\hline \multirow{6}{*}{ (i) } & & Fusarium spp. & 6 \\
\hline & & Acremonium spp. & 2 \\
\hline & & Colletotrichum dematium & 2 \\
\hline & & Total & 67 \\
\hline & & Alternaria spp. & 8 \\
\hline & & Curvularia spp. & 6 \\
\hline \multirow[t]{5}{*}{ (ii) } & \multirow[t]{5}{*}{ Dematiaceous } & Cladosporium spp. & 3 \\
\hline & & Fonsecaea spp. & 3 \\
\hline & & Total & 20 \\
\hline & & Candida albicans & 12 \\
\hline & & Candida tropicalis & 5 \\
\hline \multirow[t]{4}{*}{$B$} & \multirow[t]{3}{*}{ Yeasts } & Candida glabrata & 4 \\
\hline & & Candida parapsilosis & 9 \\
\hline & & Total & 30 \\
\hline & TOTAL & 117 & \\
\hline
\end{tabular}

\section{Discussion}

Occulomycoses or opportunistic fungal infections of the eye are increasingly recognized as important causes of morbidity and blindness, the cornea being the most frequently isolated. Studying the demographic data, total number of males were significantly higher than females indicating significant exposure of organism in their working environment and ususaaly meales are working in factories The most common organism causing fungal keratitis in this study was found to be filamentous hyaline fungi, i.e., Aspergillus spp. and predominantly Aspergillus flavus. A. flavus is isolated from soil especially in tropical \& sub-tropical areas from forage, decaying vegetation, stored seeds \& grains, damp clothing \& shoes during summer in tropics. A similar pattern was also observed by Leck AK et. al in Ghana [6].

Aspergillus can grow anywhere under a wide range of environmental conditions [17] \& is ubiquitous in nature. It is characterized by sterigmata which are characteristic of Aspergillus spp. bearing hundreds of light weight spores which can be easily disseminated by air and wind. It is thermophilic growing well at $37^{\circ} \mathrm{C}$ but can tolerate and grow at $50^{\circ} \mathrm{C}$ also. In our study relatively high incidence has been observed in dry climate and high temperatures. During summer the average temperature may reach up to $40^{\circ} \mathrm{C}$ to $42^{\circ} \mathrm{C}$ in western part of Maharashtra. Aspergillus spp. with its thermo tolerant character can survive in this environment \& can lead to fungal keratitis in patients with predisposing factors. 
Houang et. al reviewed the relation of fungal keratitis to climate concluding that, although higher incidence of fungal keratitis could be expected in countries with similar annual rainfall and temperature range, this was not always so and was also dependent on the extent of urbanization [18]. Aspergillus species were predominant in West Bangal, Mumbai, and North India, parts of South India, Nepal and Bangladesh.

Even though most cases of fungal keratitis present typical clinical presentation, there may be certain exceptional features presented by some fungal agents like Fusarium spp. It produces very severe infection with rapid onset of perforation of the cornea. Vision may be completely lost if timely therapeutic intervention is not initiated. We have isolated 6 Fusarium spp. from fungal keratitis cases. Of these, 2 were severed and lead to malignant glaucoma. Fusarium spp. have also been found to be commonest pathogen in Florida, Nigeria, Tanzania, Hongkong and Singapore. John BR. et. al and Vemuganti GK et. al also found similar findings [19]. Collectotrichum dematium was isolated from two patients and both were insulin dependent diabetes mellitus. Kaliamurthy et. al. 2004 reported parallel findings. Among the yeast and yeast like fungi implicated Candida spp. predominates. Candida albicans and Candida parapsilosis are the most frequent. Our study recorded parallel findings (12 \& 9 isolates respectively). Further 4 of Candida glabrata and 5 Candida tropicalis were also isolated. Since different species exhibit different virulence factors \& varying susceptibility to antifungal agents, it is important to identify isolates of Candida to species level. Primary treatment failure was seen I $37 \%$ of cases which is significantly higher than the earlier information, this is because of fungal keratitis were more likely to perforate, thus requiring keratoplasty more often. However the relevance of susceptibility testing to clinical outcome in keratitis cases remains unclear $[20,21,22]$.

\section{Conclusion}

The study will provide a rational approach for initial therapy, taking into account changing trends in etiology. Present study provides regional trend of fungal etiology in fungal keratitis. In conclusion, clinical suspicion by the ophthalmologists should be confirmed by microbiological finding prior to initiating corticosteroids and antibacterial antibiotics. Precise identification of the causative fungus and institution of appropriate treatment strategy could save the blindness of the eye.

\section{References}

[1] Whitcher J.P., Srinivasan M. and Upadhyay M.P. (2001) Bull. World Health Organ, 79, 214-221.

[2] Sharma S., Srinivasan M. and George C. (1993) J. Med. Microbiol., 11, 140-147.

[3] Upadhyay M.P., Karmacharya P.C., Koirala S., et al. (1991) Am. J. Opthalmol. 15, 92-99.

[4] Choi D.M., Goldstein M.H., Salierno A., et al. (2001) CLAO J. 27, 111-112.

[5] Srinivasan M., Gonzales C.A., George C., et al. (1997) Br. J. Opthalmol., 81, 965-71.

[6] Leck A.K., Thomas P.A., Hagan M., et al. (2002) Br. J. Opthalmol., 86, 1211-1215.

[7] Hagan M., Wright E., Newman M., et al. (1995) Br. J. Opthalmol., 79, 1024-1028.
[8] Coster D.J., Wilhelmus K., Peacock J., et al. (1981) International Congress and Symposium Series No. 40, 395-398.

[9] Panda A., Sharma N., Das G., et al. (1997) Cornea, 16, 2952999.

[10]Tenure M.A., Cohen E.J., Sudesh S., et al. (2000) Cornea, 19, 307-312.

[11]Gopinathan U., Garg P., Fernandes M., et al. (2002) Cornea, 21, 555-559.

[12]Thomas P.A. (2003) Clin. Microbial Rev., 16, 730-797.

[13]Froster R.K. and Rebell G. (1975) Arch Opthalmol, 93, 975978.

[14]Thomas P.A. (2003) Eye, 17, 852-862.

[15]Jones D.B., Liesegang T.J. and Robinson N.M. (1981) laboratory diagnosis of ocular infections.

[16]Merz W.G. and Roberts G.D. (1995) Manual of Clinical microbiology, 709-22.

[17]Periman L.M., Harrison D.A. and Kim J. (2003) J. Refract. Surg., 19: 364-366.

[18]Houang E., Lam D., Fan D., et. al. (2001) Trans. r soc. Trop. Med. Hyg., 95, 361-367.

[19]Venugopal P.V., Venugopal T.V., Gomathi A., et al. (1989) Indian J. Pathol. Microbiol., 32, 190-7.

[20]Anderson K.L., Mitra S., Salouli R. and Taylor H.R. (2004) Cornea, 23, 516-21.

[21]Prats C.H., Tello F.L., Jose A.B.S., et al. (2004) Ann Pharmacol. 38, 414-7.

[22]Chang C.W., Ho C.K., Chen Z.C., et al. (2002) Arch Environ Health, 57, 349-354.

[23]Dunlop A.A., Wright E.D., et al. (1994) Aust. NZ J. Ophthalm., $22,105-10$

[24]Kalimurthy J., Kalawathy C.M., et al. (2004) Cornea, 23, 3-12. 